CERN-TH/97-349

KEK-TH-550

hep-ph/9712260

\title{
Constraints on Non-Standard Contributions to the Charged-Current Interactions
}

\author{
Kaoru Hagiwara ${ }^{1,2}$ and Seiji Matsumoto ${ }^{3}$ \\ ${ }^{1}$ Theory Group, KEK, Tsukuba, Ibaraki 305, Japan \\ ${ }^{2}$ ICEPP, University of Tokyo, Hongo, Bunkyo-ku, Tokyo 113, Japan \\ ${ }^{3}$ TH Division, CERN, CH-1211 Geneva 23, Switzerland
}

\begin{abstract}
The success of the quantum level predictions of the Standard Model on the $Z$ boson properties, on $m_{W}$ and on $m_{t}$, which makes use of the muon lifetime as an input, implies a stringent constraint on new physics contributions to the $V-A$ charged-current interactions among leptons. Observed unitarity of the CKM matrix elements then implies constraints on non-standard contributions to the lepton-quark charged-current interactions. By using the recent electroweak data as inputs, we find the $95 \% \mathrm{CL}$ limits for the corresponding contact interactions: $\Lambda_{C C,+}^{\ell \ell}>7.5 \mathrm{TeV}$ and $\Lambda_{C C,-}^{\ell \ell}>10.2 \mathrm{TeV}$ for the purely leptonic terms, and $\Lambda_{C C,+}^{\ell q}>5.8 \mathrm{TeV}$ and $\Lambda_{C C,-}^{\ell q}>10.1 \mathrm{TeV}$ for the leptonquark contact interactions.
\end{abstract}

CERN-TH/97-349

December 1997 
The reports from HERA [1, 2] of event rates above Standard Model (SM) expectations in $e^{+} p \rightarrow e^{+} X$ deep inelastic scattering at very high $Q^{2}$ renewed the interest in the present constraints on non-standard interactions among leptons and quarks [3 6]. In particular, studies on low energy neutral current experiments find stringent constraints on parity-violating lepton-quark interactions [3 7], and those including high- $Q^{2}$ experiments at the Tevatron and LEP2 find constraints on general lepton-quark contact interactions in the neutral currents [5, 8]. In terms of the "compositeness scale" [9, 10], the 95\% CL lower bounds are found to be typically of the order of $10 \mathrm{TeV}$ for chiral contact terms while they can be as low as $3 \mathrm{TeV}$ for those contact terms that conserve parity or with purely axial-vector quark currents [8]. In view of the above constraints, it is unlikely that the observed excess of high- $Q^{2}$ events at HERA can be explained as a consequence of new chirality and flavour-conserving neutral currents between leptons and quarks.

As for charged-current (CC) $e^{+} p \rightarrow \bar{\nu}_{e} X$ events, both H1 and ZEUS experiments observed about a factor of 2 more events than expected in the SM at $Q^{2}>10,000 \mathrm{GeV}^{2}$ [1, 11]. Although the statistics of high- $Q^{2}$ events is still limited, one may hope to probe new interactions at HERA also in the charged currents 12 18].

In this letter, we would like to point out that non-standard contributions to the CC interactions are strongly constrained by the electroweak measurements, which are consistent with the predictions of the minimal SM and also by the observed unitarity of the Cabibbo-Kobayashi-Maskawa (CKM) matrix elements. Normally, one takes as inputs the observed muon decay constant $G_{F}$, the $Z$ boson and the top-quark masses, and an estimate of the running QED coupling $\alpha\left(m_{Z}^{2}\right)$. The predictions are made for all the other electroweak observables, including various asymmetries on the $Z$ pole and the $W$ mass, as functions of an assumed Higgs boson mass $m_{H}$. We show that by using all the electroweak data as inputs besides $G_{F}$ we can calculate the muon decay constant in the 
minimal SM, and that by comparing it with the observed value we can obtain constraints on non-SM contributions to the leptonic charged currents. The observed unitarity of the CKM matrix elements then constrains the difference between new physics contributions to the purely leptonic and lepton-quark CC interactions.

Effects of new physics at energies below new particle thresholds can generally be expressed as non-renormalizable higher-dimensional terms in the effective Lagrangian of the SM particles. The effective CC interactions among quarks and leptons may be parametrized as

$$
\mathcal{L}_{C C}=\sum_{f_{i}} \sum_{\alpha, \beta} \eta_{\alpha \beta}^{f_{1} f_{2} f_{3} f_{4}} S \overline{\psi_{f_{1}}} \gamma^{\mu} P_{\alpha} \psi_{f_{2}} \overline{\psi_{f_{3}}} \gamma_{\mu} P_{\beta} \psi_{f_{4}}
$$

where $\left(f_{1}, f_{2}\right)$ and $\left(f_{3}, f_{4}\right)$ are lepton and/or quark charged currents, $\alpha, \beta=L, R$ denote their chirality: $P_{L}=\left(1-\gamma_{5}\right) / 2, P_{R}=\left(1+\gamma_{5}\right) / 2$, and $S$ is a statistical factor, which is $1 / 2$ if the two currents are the same and is otherwise 1 . The coefficients $\eta_{\alpha \beta}^{f_{1} f_{2} f_{3} f_{4}}$ have the dimension of (mass) ${ }^{-2}$, which may be expressed as 9,10

$$
\eta_{\alpha \beta}^{f_{1} f_{2} f_{3} f_{4}}=\epsilon \frac{4 \pi}{\left(\Lambda_{\alpha \beta, \epsilon}^{f_{1} f_{2} f_{3} f_{4}}\right)^{2}},
$$

with the sign factor $\epsilon=+$ or - . If the contact interactions are results of an exchange of an extra heavy charged vector boson $W_{E}$, they are given by

$$
\eta_{\alpha \beta}^{f_{1} f_{2} f_{3} f_{4}}=-\frac{g_{\alpha}^{f_{1} f_{2}} g_{\beta}^{f_{3} f_{4}}}{m_{W_{E}}^{2}},
$$

where $g_{\alpha}^{f_{1} f_{2}}$ and $g_{\beta}^{f_{3} f_{4}}$ are the $W_{E}$-boson couplings to the corresponding lepton and quark currents.

In this report we study only the $V-A$ contact interactions, $\alpha=\beta=L$ terms in eq. (1), because they interfere with the SM amplitudes and because, without light right-handed neutrinos, $V+A$ currents do not contribute to low energy leptonic or lepton-quark CC processes. Also, we do not consider mixing between the SM $W$ boson and possible additional $W_{E}$ bosons. The latter assumption 
allows us to use the observed $W$ mass in the SM contributions to the muon decay amplitude.

Then, the observed Fermi constant $G_{F}$ is expressed as

$$
\begin{aligned}
4 \sqrt{2} G_{F} & =\frac{\bar{g}_{W}^{2}(0)+\hat{g}^{2} \bar{\delta}_{G}}{m_{W}^{2}}-\eta_{L L}^{e \nu_{e} \nu_{\mu} \mu} \\
& \approx \frac{\bar{g}_{W}^{2}(0)}{m_{W}^{2}}\left[1+\bar{\delta}_{G}-\frac{\eta_{L L}^{e \nu_{e} \nu_{\mu} \mu}}{4 \sqrt{2} G_{F}}\right] .
\end{aligned}
$$

Here $\bar{g}_{W}^{2}(0)$ and $\bar{\delta}_{G}$ are the universal charge form factor and the radiative corrections coming from vertex and box diagrams to the $\mu$-decay, respectively. The hatted symbols, $\hat{s}$ and $\hat{g}$, denote $\overline{\mathrm{MS}}$ couplings (see Ref. 119 for the notation). From eq. (4), we see immediately that the effect from the new contact term $\eta_{L L}^{e \nu_{e} \nu_{\mu} \mu}$ can be regarded as a non-standard contribution to the parameter $\bar{\delta}_{G}$. In Ref. [19], it has been shown that only a combination $T^{\prime}$ such that

$$
T^{\prime}=T+\frac{\bar{\delta}_{G}^{\mathrm{SM}}-\bar{\delta}_{G}}{\alpha}
$$

is constrained by the electroweak experiments in the presence of non-standard contributions to $\bar{\delta}_{G}$. Here the parameter $T$ [21] is a measure of the difference between the neutral- and charged-current interactions at low energies. The effect of the new contact interaction can hence be constrained by the difference $\Delta T$ between the measured $T^{\prime}$ parameter and the SM prediction for the $T$ parameter:

$$
\Delta T=T^{\prime}-T_{\mathrm{SM}}=\frac{\eta_{L L}^{e \nu_{e} \nu_{\mu} \mu}}{4 \sqrt{2} G_{F} \alpha}
$$

In the following analysis we use recent electroweak datal of the $Z$ parameter measurements at LEP1 and SLC [22] and the $W$ mass measurements at the Tevatron and LEP2 [22]. In addition, we use the combined value of the topquark mass measured at CDF and D0, $m_{t}=175.6 \pm 5.5 \mathrm{GeV}$ 22], the QCD coupling estimated by PDG, $\alpha_{s}=0.118 \pm 0.003$ [10], and the estimate of the

\footnotetext{
${ }^{1}$ We do not include the low energy neutral current (LENC) data (see Ref. [ / in ) in letter because they may be affected by the presence of new contact interactions in the neutral current sectors. The properties of the $Z$ and $W$ bosons on the pole are not affected by the new interactions as long as the mixing between the SM weak bosons and the new vector bosons are negligible.
} 
QED coupling at the $m_{Z}$ scale, $\delta_{\alpha} \equiv 1 / \bar{\alpha}\left(m_{Z}^{2}\right)-128.72=0.03 \pm 0.09$ 24. We calculate all radiative corrections within the SM, except that we allow a new contribution $\Delta T$ to the $T^{\prime}$ parameter (see Refs. [19,20] for details).

From the fit to the $Z$ boson parameters and the $W$ mass, we obtain

$$
\Delta T \equiv T^{\prime}-T_{\mathrm{SM}}=-0.06_{-0.11}^{+0.26} \text { or } \eta_{L L}^{e \nu_{e} \nu_{\mu} \mu}=\left(-0.03_{-0.05}^{+0.13}\right) \mathrm{TeV}^{-2} .
$$

as the $1 \sigma$ constraints. In Fig.1, we show the minimal $\chi^{2}$ as a function of $\Delta T$, where the Higgs boson mass $m_{H}$ is allowed to vary in the fit. The solid line is obtained by constraining $m_{H}$ in the range $77.1 \mathrm{GeV}<m_{H}<1 \mathrm{TeV}$ : the lower limit is from direct measurements at LEP2 [23] while our perturbative calculation is unreliable at $m_{H} \gtrsim 1 \mathrm{TeV}$.

For comparison, we also give the result obtained by allowing $m_{H}$ to vary freely, without the boundaries. As seen from the figure, both results are the same for $-0.1 \lesssim \Delta T \lesssim 0.35$ because the fitted $m_{H}$ value stays in the allowed region. On the other hand, for $\Delta T \lesssim-0.1$, the best fit value of $m_{H}$ is found below $77.1 \mathrm{GeV}$ for the dashed line. This explains the difference between the two curves. The constraint (7) and all the following results are obtained by requiring $77.1 \mathrm{GeV}<m_{H}<1 \mathrm{TeV}$.

In order to obtain the $95 \%$ confidence level limit for $\eta$, we assume that the probability density function $P(\eta)$ is proportional to $\exp \left(-\chi^{2}(\eta) / 2\right)$. Further we need to consider two cases separately to convert the limit for $\eta$ to that for $\Lambda$ [8]. The $95 \%$ CL limit on $\Lambda_{+}$, which corresponds to $\epsilon=+1$ in eq. (2), is obtained from $\eta_{+}=4 \pi / \Lambda_{+}^{2}$, where

$$
0.05=\frac{\int_{\eta_{+}}^{\infty} P(\eta) d \eta}{\int_{0}^{\infty} P(\eta) d \eta}
$$

Likewise the $95 \%$ CL limit on $\Lambda_{-}$is obtained from $\eta_{-}=-4 \pi / \Lambda_{-}^{2}$, where

$$
0.05=\frac{\int_{-\infty}^{\eta_{-}} P(\eta) d \eta}{\int_{-\infty}^{0} P(\eta) d \eta} .
$$


We find the 95\% CL limits for $\Lambda_{ \pm}$月:

$$
\begin{aligned}
& \Lambda_{L L,+}^{e \nu_{e} \nu_{\mu} \mu} \equiv \Lambda_{C C,+}^{l l}>7.5 \mathrm{TeV} \\
& \Lambda_{L L,-}^{e \nu_{e} \nu_{\mu} \mu} \equiv \Lambda_{C C,-}^{l l}>10.2 \mathrm{TeV}
\end{aligned}
$$

Next we consider the limit on the lepton-quark contact interactions. We assume the unitarity of the CKM in the SM:

$$
\left|V_{u d}^{\mathrm{SM}}\right|^{2}+\left|V_{u s}^{\mathrm{SM}}\right|^{2}+\left|V_{u b}^{\mathrm{SM}}\right|^{2}=1
$$

Experimentally, this unitarity is slightly violated at the $1.7 \sigma$ level [10]:

$$
\left|V_{u d}\right|^{2}+\left|V_{u s}\right|^{2}+\left|V_{u b}\right|^{2}=0.9965 \pm 0.0021
$$

In the presence of new contact interactions in the CC sector, the observed CKM matrix elements can be shifted as

$$
V_{u d_{j}}^{\mathrm{obs}}=V_{u d_{j}}^{\mathrm{SM}}-\frac{\eta_{L L}^{\ell \nu_{\ell} u d_{j}}-V_{u d_{j}}^{\mathrm{SM}} \eta_{L L}^{e \nu_{e} \nu_{\mu} \mu}}{4 \sqrt{2} G_{F}}
$$

because the CKM matrix elements are experimentally determined from the ratio of the semileptonic and the purely leptonic CC strengths ${ }^{3}$.

In general, three lepton-quark contact interactions in the $V-A$ charged current, $\eta_{L L}^{\ell_{\nu} u d}, \eta_{L L}^{\ell_{\ell} u s}, \eta_{L L}^{\ell_{\ell} u b}$, can enter the unitarity constraint (12). By assuming e.g. $\eta_{L L}^{\ell \nu_{\ell} u s}=\eta_{L L}^{\ell \nu_{\ell} u b}=0$, one can obtain constraint on $\eta_{L L}^{\ell \nu_{\ell} u d}-V_{u d} \eta_{L L}^{e \nu_{e} \nu_{\mu} \mu}$. In this paper, we examine the case where the contact interactions satisfy the $\mathrm{SU}(2)_{\mathrm{L}} \times \mathrm{U}(1)_{\mathrm{Y}}$ gauge symmetry of the SM. Then $V-A$ charged-current interactions among leptons and those between leptons and quarks are expressed in terms of left-handed lepton doublets, $L_{\ell}=\left(\nu_{\ell}, \ell_{L}\right)(\ell=e, \mu, \tau)$, and quark doublets $Q_{i}=\left(u_{i L}, d_{i L}\right)(i=1,2,3)$. Assuming that the new interactions do

\footnotetext{
${ }^{2}$ In order to test the sensitivity of the above bounds to the estimate of $\bar{\alpha}\left(m_{Z}^{2}\right)$, we repeat the analysis by using the estimate $\delta_{\alpha}=0.12 \pm 0.06$ 25]. We find $\Lambda_{+}>7.8 \mathrm{TeV}$ and $\Lambda_{-}>9.7 \mathrm{TeV}$ as the $95 \%$ CL limits, which donot differ much from the results (10) that are obtained with the estimate $\delta_{\alpha}=0.03 \pm 0.09$ 24.

${ }^{3}$ By setting $\eta_{L L}^{e \nu_{e} \mu}=0$, the formula 13 ) reduces to that of Altarelli et.al. 18 who neglect contact interactions to the purely leptonic channel.
} 
not distinguish between quark and lepton generations, CC contact interactions should have the following universal form [8]

$$
\mathcal{L}_{\mathrm{SU}(2)}=\frac{\eta_{2}^{L L}}{1+\delta_{i j}}\left(\overline{L_{i}} \gamma^{\mu} T^{a} L_{i}\right)\left(\overline{L_{j}} \gamma_{\mu} T^{a} L_{j}\right)+\eta_{2}^{L Q}\left(\overline{L_{i}} \gamma^{\mu} T^{a} L_{i}\right)\left(\overline{Q_{j}} \gamma_{\mu} T^{a} Q_{j}\right)
$$

Here summation of $i, j$ indices over the three generations is understood. Among the 4 purely leptonic and 7 lepton-quark contact terms in the most general effective Lagrangian [8], only the above two terms with the $\mathrm{SU}(2)$ generators $T^{a}=\sigma^{a} / 2$ contribute to the CC processes. We find

$$
\eta_{L L}^{\ell \nu_{\ell} \nu_{\ell^{\prime}} \ell^{\prime}}=\frac{1}{2} \eta_{2}^{L L}
$$

for the purely leptonic terms and

$$
\eta_{L L}^{\ell \nu_{\ell} u_{i} d_{j}}=V_{u_{i} d_{j}}^{\mathrm{SM}} \frac{1}{2} \eta_{2}^{L Q}
$$

for the lepton-quark interactions in terms of the quark mass eigenstates, where $V_{u_{i} d_{j}}^{\mathrm{SM}}$ are the standard CKM matrix elements.

By inserting

$$
V_{u d_{j}}^{\mathrm{obs}}=V_{u d_{j}}^{\mathrm{SM}}\left[1-\frac{1}{2} \frac{\eta_{2}^{L Q}-\eta_{2}^{L L}}{4 \sqrt{2} G_{F}}\right]
$$

into eq. (12) and using the unitarity relation of the SM, eq. (11), we obtain

$$
\frac{1}{2}\left[\eta_{2}^{L Q}-\eta_{2}^{L L}\right]=(0.116 \pm 0.069) \mathrm{TeV}^{-2}
$$

As expected, there is a $1.7 \sigma$ signal in the difference of the lepton-quark and purely leptonic CC contact interactions. If one assumes $\eta_{2}^{L L}=0$ [18], the above result (18) constrains $\eta_{2}^{L Q}$. On the other hand, if we allow $\eta_{2}^{L L}$ to vary freely under the constraint (7), we find

$$
\frac{1}{2} \eta_{2}^{L Q}=\epsilon \frac{4 \pi}{\left(\Lambda_{C C, \epsilon}^{\ell q}\right)^{2}}=\left(0.09_{-0.09}^{+0.14}\right) \mathrm{TeV}^{-2} .
$$

By studying the probability distributions in the region of $\epsilon>0$ and $\epsilon<0$ separately, as in eqs. (8) and (9), we obtain the following 95\% CL bounds:

$$
\begin{aligned}
& \Lambda_{C C,+}^{\ell q}>5.8 \mathrm{TeV}, \\
& \Lambda_{C C,-}^{\ell q}>10.1 \mathrm{TeV} .
\end{aligned}
$$


Here we allow $\eta_{2}^{L L}$ to vary freely, taking both signs. If we assume no new physics contribution to the purely leptonic charged currents, $\eta_{2}^{L L}=0$, then the constraint (18) gives

$$
\begin{aligned}
& \Lambda_{C C,+}^{l q}>7.4 \mathrm{TeV} \quad(95 \% \mathrm{CL}), \\
& \Lambda_{C C,-}^{l q}>12.4 \mathrm{TeV} \quad(95 \% \mathrm{CL}) .
\end{aligned}
$$

The above constraints (21) differ significantly from the ones quoted in 18], because we obtained the bounds in the $\epsilon>0$ and $\epsilon<0$ regions separately. In view of the above constraint (19), deviation from the SM in the CC cross section

at $Q^{2} \sim 15,000 \mathrm{GeV}^{2}$ due to $V-A$ contact interactions should be at the level of at most one per cent.

\section{Acknowledgements}

The authors wish to thank K. Tokushuku for calling our attention to the chargedcurrent data at HERA, and V. Barger and D. Zeppenfeld for enlightening discussions. They also wish to thank S. Vascotto for careful reading of the manuscript. This research was supported in part by the JSPS-NSF Joint Research Project. The work of S.M. is supported by the CERN-Asia Fellowship.

\section{References}

[1] The H1 Collaboration, C. Adloff et al., Z. Phys. C74, 191 (1997).

[2] The ZEUS Collaboration, J. Breitweg et al., Z. Phys. C74, 207 (1997).

[3] G. Altarelli, J. Ellis, G.F. Giudice, S. Lola, and M.L. Mangano, preprint CERN-TH/97-040 hep-ph/9703276.

[4] K.S. Babu, C. Kolda, J. March-Russell, and F. Wilczek, Phys. Lett. B402, 367 (1997). 
[5] V. Barger, K. Cheung, K. Hagiwara, and D. Zeppenfeld, Phys. Lett. B404, 147 (1997).

[6] A. Nelson, Phys. Rev. Lett. 78, 4159 (1997); N. Bartolomeo and M. Fabbrichesi, Phys. Lett. B406, 237 (1997); M.C. Gonzalez-Garcia and S.F. Novaes, hep-ph/9703346; W. Buchmüller and D. Wyler, hep-ph/9704317; S. Godfrey, Mod. Phys. Lett. A12, 1859 (1997); N.G. Deshpande, B. Dutta, and Xiao-Gang He, hep-ph/9705236; L. Giusti and A. Strumia, hepph/9706298; Z. Cao, X.-G. He, and B. McKellar, hep-ph/9707227.

[7] G.-C. Cho, K. Hagiwara and S. Matsumoto, hep-ph/9707334.

[8] V. Barger, K. Cheung, K. Hagiwara, and D. Zeppenfeld, hep-ph/9707412.

[9] E. Eichten, K. Lane, and M. Peskin, Phys. Rev. Lett. 50, 811 (1982).

[10] Review of Particle Properties, Phys. Rev. D54, 1 (1996).

[11] B. Straub, talk presented at Lepton-Photon '97 Symposium, Hamburg, 1997

E. Elsen, International Europhysics Conference on High Energy Physics (EPS97) 19-26 August 1997, Jerusalem, Israel.

[12] R. Rückl, Phys. Lett. B129, 363 (1983); Nucl. Phys. B234, 91 (1984).

[13] C. J. C. Burges and H. J. Schnitzer, Phys. Lett. 134B, 329 (1984).

[14] R.J. Cashmore et al., Phys. Rev. 122, 275 (1985).

[15] C.P. Burgess, S Godfrey, H Konig, D. London, I. Maksymyk, Phys. Rev. D49, 6115 (1994).

[16] P. Chiappetta and J.-M. Virey, Phys. Lett. B389, 89 (1996).

[17] F. Cornet and J. Rico, Phys. Lett. B412, 343 (1997). 
[18] G. Altarelli, G.F. Giudice, and M.L. Mangano, preprint CERN-TH/97-101 hep-ph/9705287.

[19] K. Hagiwara, D. Haidt, C.S. Kim and S. Matsumoto, Z. Phys. C64, 559 (1994); Errata C68, 352 (1995).

[20] K. Hagiwara, D. Haidt and S. Matsumoto, hep-ph/9706331, to be published in Z. Phys. C (1997).

[21] M.E. Peskin and T. Takeuchi, Phys. Rev. Lett. 65, 964 (1990); Phys. Rev. D46, 381 (1992).

[22] D. Ward, talk presented at International Europhysics Conference on High Energy Physics (EPS97), 19-26 August 1997, Jerusalem, Israel.

[23] P. Janot, talk presented at International Europhysics Conference on High Energy Physics (EPS97), 19-26 August 1997, Jerusalem, Israel.

[24] S. Eidelman and F. Jegerlehner, Z. Phys. C67, 585 (1995).

[25] A.D. Martin and D. Zeppenfeld, Phys. Lett. B345, 558 (1995). 


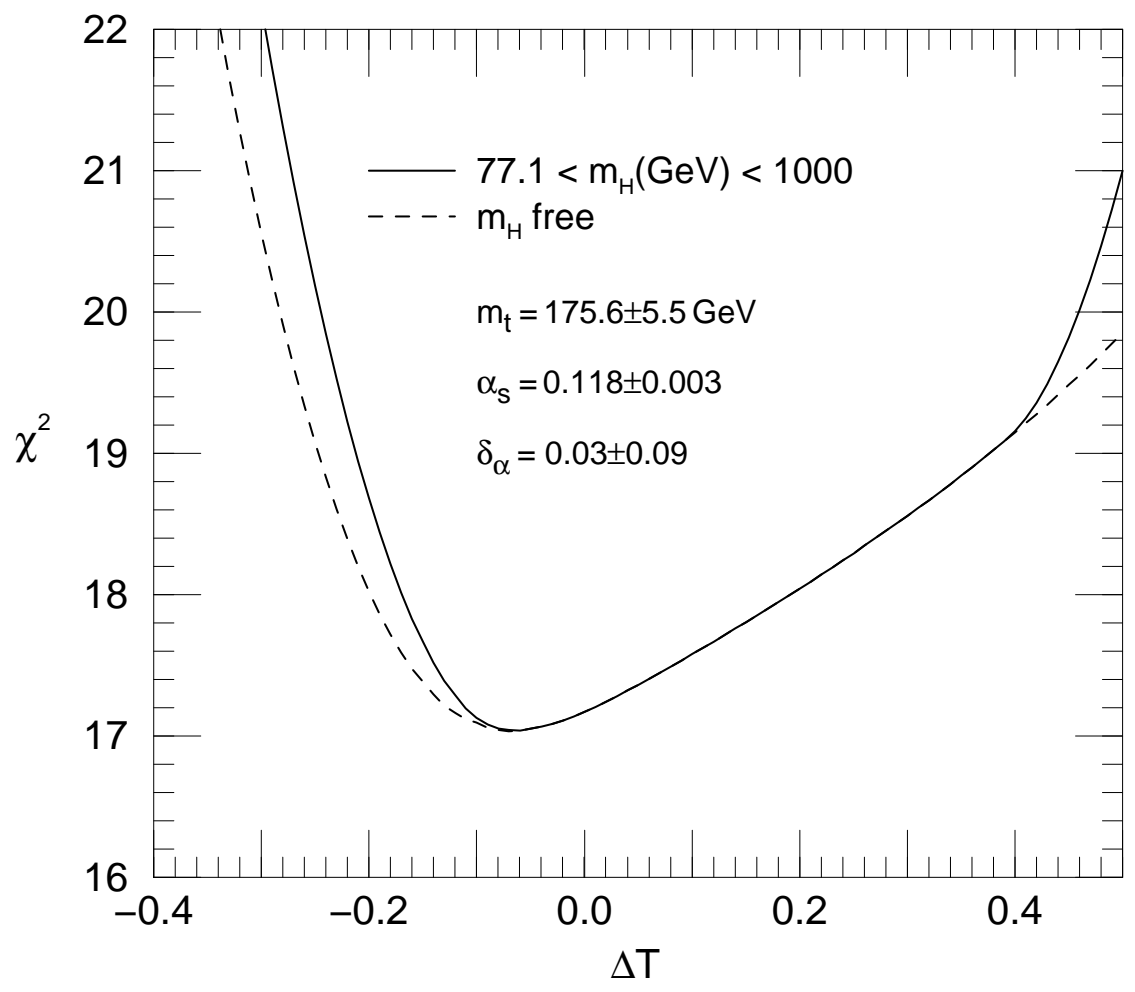

Figure 1: The minimal $\chi^{2}$ versus $\Delta T$ when $m_{H}$ is allowed to vary. The solid line is obtained by constraining $m_{H}$ in the range $77.1 \mathrm{GeV}<m_{H}<1 \mathrm{TeV}$ while the dashed line is obtained by allowing $m_{H}$ to vary freely. 